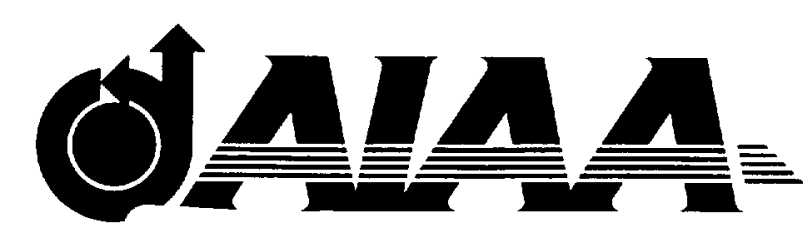

NASA-TM-112834

AIAA 97-1581

Continued Development of the Discontinuous Galerkin Method for Computational Aeroacoustic Applications H.L. Atkins

NASA Langley Research Center Hampton, VA 23681

3nd AIAA/CEAS Aeroacoustics Conference May 12-14, 1997 / Atlanta, GA 
- 


\title{
Continued Development of the Discontinuous Galerkin Method for Computational Aeroacoustic Applications
}

\author{
H. L. Atkins* \\ NASA Langley Research Center \\ Hampton, VA, 23681-0001
}

\begin{abstract}
The formulation and the implementation of boundary conditions within the context of the quadrature-free form of the discontinuous Galerkin method are presented for several types of boundary conditions for the Euler equations. An important feature of the discontinuous Galerkin method is that the interior point algorithm is well behaved in the neighborhood of the boundary and requires no modifications. This feature leads to a simple and accurate treatment for wall boundary conditions and simple inflow and outflow boundary conditions. Curved walls are accurately treated with only minor changes to the implementation described in earlier work. The "perfectly matched layer" approach to nonreflecting boundary conditions is easily applied to the discontinuous Galerkin. The compactness of the discontinuous Galerkin method makes it better suited for buffer-zone-type methods than high-order finite-difference methods. Results are presented for wall, characteristic inflow and outflow, and nonreflecting boundary conditions.
\end{abstract}

\section{Introduction}

Much of the recent work in computational aeroacoustics (CAA) has focused on improvements to traditional finite-difference methods to increase the accuracy and to implement specialized boundary conditions. While this approach has promoted a rapid growth of the field, these methods place constraints on the mesh smoothness that make their application to highly complex geometries problematic. Furthermore, the improved spatial operators are not applicable in the neighborhood of some critical flow phenomenon, such as shock waves, with out substantial modifications. The goal of this work is to develop

Copyright (C) 1997 by the American Institute of Aeronautics and Astronautics, Inc. No copyright is asserted in the United States under Title 17, U.S. Code. The U.S. Government has a royalty-free license to exercise all rights under the copyright claimed herein for government purposes. All other rights are reserved by the copyright owner.

"Senior member, AIAA robust and efficient methods that jive accurate solutions independent of grid smoothness.

The discontinuous Galerkin meihod is a highly compact formulation that provides a method of obtaining the high accuracy required or CAA on nonsmooth unstructured grids. The bility to use an unstructured grid greatly simplifies the largest obstacle in computing the flow arounc complex geometries: the generation of the grid. $I_{1}$. reference 1 , the discontinuous Galerkin method wis formulated in a quadrature-free form that reduc $\cdots$ d the computational effort and storage requiremer.ts. In that work, the method was described in detail along with basic benchmark cases that demonstrate the accuracy and robustness of the method for the scalar advection equation and for the linear Euler $\approx$ quations. That work focused on the new implementation of the interior point scheme and addressed only periodic domains.

In this article, the formulation and implementation of several types of boundary conditions for the linear Euler equations are describet. Also discussed are features of the discontinuous salerkin method that make the application of boundary conditions relatively straightforward and robust. These beneficial features are all attributable to the inherent compactness of the discontinuous (alerkin method.

Most methods used for CA $\mathrm{A}$ today fall in the category of high-order finite difference methods such, as the widely used di-persion-relationpreserving (DRP) scheme. ${ }^{2}$ Effort to develop specialized boundary conditions for j:roblems particular to aeroacoustics have focused o: finite-difference methods, but much of the work is tlso applicable to the discontinuous Galerkin methoul. In some cases, such as the work on wall bound ry conditions by Tam and Dong, ${ }^{3}$ special boundary conditions are needed to counter errors associaled with the application of finite-difference methods near a boundary: errors that do not occur in :he discontinuous Galerkin method.

The most problematic boundary in CAA is the boundary that is produced when ai infinite or semi- 
infinite domain is truncated to a finite computational domain. In this case, precise flow conditions are not known at the boundary of the computational domain, and the boundary condition becomes more of a goal than a precise mathematical statement. In particular, the boundary condition seeks to make the flow field behave as if the computational domain were larger; waves are allowed to exit the computational domain with no nonphysical side affects. In CAA, the boundary conditions appropriate for this type of boundary are referred to as nonreflecting boundary conditions.

Boundary conditions used for steady and unsteady aerodynamic calculations have relied primarily on characteristic formulations, such as the simple relations proposed by Jameson et al. ${ }^{4}$ to ensure that the correct information enters and leaves the domain; however, these methods become less accurate as the size of the computational domain is reduced. Efforts to improve on this have taken many forms, which range from efforts to analytically solve a simplified equation in the infinite domain outside the computational domain ${ }^{5}, 6$ to methods that solve specialized equations at the boundary or in a small region near the boundary. $2,7,8,9,10,11$ The methods work well when an acoustic wave exits the domain normal to the boundary; however, in other cases these methods produce predictable reflections that depend on the angle of incidence in a manner that is fairly well understood in most cases.

Two exceptions to this are the perfectly matched layer (PML) method of $\mathrm{Hu}^{10}$ and the asymptotic method of Hagstrom ${ }^{8}$ and Goodrich and Hagstrom. ${ }^{11}$ Hagstrom's approach is similar to that of Engquist and Majda ${ }^{7}$ Giles, ${ }^{9}$ and many others, except that the use of a Padé series approximation leads to a convergent sequence of equations in which the error associated with the wave incidence is reduced as more terms are retained. In the PML approach, a split and damped form of the governing equations is solved in a finite region near the boundary. Under certain constraints, no reflection of a wave of any incidence occurs at the interface between the main computational domain and the region where the PML method is applied. Within the PML region, waves are damped such that any reflection of the wave off the outer boundary of the PML zone is insignificant. Because of the compact nature of the discontinuous Galerkin method, the PML method is more easily implemented for the discontinuous Galerkin method than for finite-difference methods.

The first section of this article briefly describes the discontinuous Galerkin method and the quadrature- free form of the implementation; the reader is referred to reference 1 for complete details. The second section describes issues related to boundary conditions and outlines the general approach to applying boundary conditions. The remaining sections deal with special issues of curved-wall and nonreflecting boundary conditions. Treatment of curved walls requires a minor modification to the basic formulation. Two types of nonreflecting boundary conditions are presented: a simple characteristic approach and the PML method.

\section{Discontinuous Galerkin Method}

The discontinuous Galerkin method is applicable to systems of first-order equations of the form

$$
\frac{\partial U}{\partial t}+\nabla \cdot \vec{F}(U)=0
$$

defined on some domain $\bar{\Omega}$ with a boundary $\partial \bar{\Omega}$, where $U=\left\{u_{0}, u_{1}, \ldots\right\}$ and $\vec{F}=\left\{\vec{f}_{0}, \vec{f}_{1}, \ldots\right\}$. The domain is partitioned into a set of nonoverlapping elements $\Omega_{i}$ that cover the domain $\bar{\Omega}=\underset{\forall i}{\cup} \Omega_{i}$. Within each element, the following set of equations is solved:

$$
\begin{aligned}
\int_{\Omega_{i}} b_{k} \frac{\partial V_{i}}{\partial t} J_{i} d \Omega & -\int_{\Omega_{i}} \nabla b_{k} \cdot \mathbf{J}_{i}^{-1} \vec{F}_{i}(V) J_{i} d \Omega \\
& +\int_{\partial \Omega_{i}} b_{k} \mathbf{J}_{i}^{-1} \vec{F}^{R} \cdot J_{i} d \vec{s}=0
\end{aligned}
$$

for $k=1,2, \ldots, N$, where $\left\{b_{k}, k=1,2, \ldots, N\right\}$ is a set of basis functions,

$$
U \approx V_{i}=\sum_{j=1}^{N} v_{i, j} b_{j}, \quad \mathbf{J}_{i}=\frac{\partial(x, y, z)}{\partial(\xi, \eta, \zeta)}
$$

and $J_{i}=\left|\mathbf{J}_{i}\right|$. Equation set (2) is obtained by projecting equation (1) onto each member of the basis set and then integrating by parts to obtain the weak conservation form. In the present work, the basis set is the set of polynomials that are defined local to the element and are of degree $\leq n$. In two dimensions, for example, the basis set is $\left\{1, \xi, \eta, \xi^{2}, \xi \eta, \eta^{2}, \ldots\right\}$ where $(\xi, \eta)$ are the local coordinates. The solution $U$ is approximated as an expansion in terms of the basis functions; thus, both $V$ and $\vec{F}$ are discontinuous at the boundary between adjacent elements (hence, the name discontinuous Galerkin). The discontinuity in $V$ between adjacent elements is treated with an approximate Riemann flux, which is denoted $\vec{F}^{R} ; \mathbf{J}_{i}$ is the Jacobian of the transformation from the global coordinates $(x, y, z)$ to the element coordinates $(\xi, \eta, \zeta)$ of element $i$. Research has shown ${ }^{12}$ 
that the upwind bias provided by the Riemann flux plays an important role in ensuring the stability of the discontinuous Galerkin method.

In the usual implementation of the discontinuous Galerkin method,' the integrals are evaluated with quadrature formulas. This approach is problematic for even moderately high-order implementations in multidimensions and has limited most efforts to $n=2$ or 3 . The difficulties arise in part because the number of quadrature points in multidimensional formulas of the required accuracy usually exceeds $N$ (the number of terms in the expansion) by a considerable margin. In the quadrature-free form, the integral evaluations are reduced to a summation over the coefficients of the solution expansion, which is an operation of order $N$. To implement the quadrature-free approach, the flux $\vec{F}$ must also be written as an expansion in terms of basis functions:

$$
\vec{F}(U) \approx \vec{G}\left(V_{i}\right)=\sum_{j=1}^{N} \vec{g}_{j}\left(V_{i}\right) b_{j}
$$

(a similar expansion is made for the approximate Riemann flux $\vec{F}^{R}$.) This procedure is trivially accomplished for linear equations, such as those of interest here. Several approaches to treating nonlinear equations are discussed and demonstrated in reference 1 . With the additional assumption that $J_{i}$ and $\mathbf{J}_{\boldsymbol{i}}$ are constant within each element, the integrals can be evaluated exactly, and the equation set can be rewritten in matrix form as

$$
\begin{array}{r}
J_{i} \mathbf{M}_{i} \frac{\partial \mathbf{V}_{i}}{\partial t}-\overrightarrow{\mathbf{A}}_{i} \cdot J_{i} \mathbf{J}_{i}^{-1} \overrightarrow{\mathbf{G}}_{i} \\
+\sum_{k=1}^{m_{1}} \mathbf{B}_{i, k}\left(J_{i} \mathbf{J}_{i}^{-1} \overrightarrow{\mathbf{G}}_{i, k}^{R}\right) \cdot \vec{s}_{i, k}=0
\end{array}
$$

where $m_{i}$ is the number of sides around element $i$, $\vec{s}_{i, k}$ is the outward unit normal on side $k, \mathbf{V}_{\mathbf{i}}=$ $\left[v_{i, 0}, v_{i, 1}, \ldots\right]^{T}, \overrightarrow{\mathbf{G}}_{i}=\left[\vec{g}_{i, 0}, \vec{g}_{i, 1}, \ldots\right]^{T}$, and $\overrightarrow{\mathbf{G}}_{i, k}^{R}=$ $\left[\vec{g}_{i, k, 0}^{R}, \vec{g}_{i, k, 1}^{R}, \ldots\right]^{T}$. The mass matrix $\mathbf{M}_{i}$ and the vector matrix $\overrightarrow{\mathbf{A}}_{i}$ are $N \times N$ matrices given by

$$
\begin{gathered}
\mathbf{M}_{i}=\left[m_{k, l}\right], \quad \overrightarrow{\mathbf{A}}_{i}=\left[\vec{a}_{k, l}\right] \\
m_{k, l}=\int_{\Omega_{i}} b_{k} b_{l} d \Omega, \quad \vec{a}_{k, l}=\int_{\Omega,} b_{l} \nabla b_{k} d \Omega
\end{gathered}
$$

for $1 \leq k, l \leq N$.

Derivation of the boundary integral terms is complicated only by the fact that the solutions on either side of the element boundary are represented in terms of different coordinate systems. This problem is resolved by expressing the solution on both sides of the element boundary in te: ms of a common edge-based coordinate system (a simple coordinate transformation). This allows the boundary integral to be expressed in terms of an edge Inatrix $\mathbf{B}_{i, k}$ times a vector that is composed of the coefficients of the approximate Riemann flux expresse $d$ in terms of the edge-based coordinate system $\overrightarrow{\mathrm{G}}_{i, i}^{R}$ (instead of the local element coordinate system $s$ in the case of $\overrightarrow{\mathbf{G}}_{i}$ ).

In addition to the requirement that $J_{i}$ and $\mathbf{J}_{\mathbf{i}}$ be constants within the element, $n$ ost elements are constrained to shapes that map into one of a few fixed simple computational elemen!s (such as a unit square or an equilateral triangle in wo dimensions). With this last constraint, the matices $\mathbf{M}_{i}, \overrightarrow{\mathbf{A}}_{i}$ and $\mathbf{B}_{i, k}$ are the same for all elements of a given type, and the products $\mathbf{M}^{-1} \overrightarrow{\mathbf{A}}$ and $\mathbf{M}^{-1} \overrightarrow{\mathbf{B}}_{k}$ can be precomputed and stored at a consid rable savings in terms of both computer storage ard computational time. This constraint is only to facilitate an efficient implementation and can be relaxe: $/$ at selected elements if the need arises (e.g., to truat curved walls). A detailed derivation of the matrices $\mathbf{M}, \overrightarrow{\mathbf{A}}$, and $\mathbf{B}_{k}$ is given in reference 1 . The syecial case of elements with curved sides is describ d in a later section. Because equation ( 3 ) is of tlie same form for all elements, the element index $i$ will be dropped for clarity.

Equation (3) is advanced in time by using the three-stage Runge-Kutta method of Shu and Osher. ${ }^{16}$ Analysis of the stability of this approach can be found in reference 1 .

\section{General Features of Bounda y Conditions}

The first two terms of equation (:) depend only on the solution within the element, and communication between adjacent elements occurs only through the Riemann flux $\overrightarrow{\mathrm{G}}^{R}$. An important ature of the discontinuous Galerkin method is that the approximate Riemann flux is the only mechanism through which an element communicates with it surroundings, regardless of whether the element buundary is in the interior of the domain or coincides with the domain boundary. A notable consequence is that the usual interior algorithm is valid in elereents adjacent to the boundary. In contrast, the interior point operator of most high-order finite-diffr-rence and finitevolume methods cannot be applied at points near the boundary without some modifications. These modifications usually result in redu:ed accuracy, and careful attention is required to prevent the introduction of instabilities. ${ }^{17}$ Thus, by use of the discontinuous Galerkin method, a major soince of error com- 
mon to many high-order finite-difference and finitevolume methods is completely avoided.

Because each element communicates with its neighbors only through the approximate Riemann flux, most boundary conditions will be imposed via the approximate Riemann flux. In this respect, the imposition of boundary conditions for the discontinuous Galerkin method is quite similar to that of low-order finite-volume methods. This similarity is especially true for the quadrature-based discontinuous Galerkin method, in which the approximate Riemann flux is evaluated at discrete boundary points and then numerically integrated. In the quadraturefree form of the discontinuous Galerkin method, the approximate Riemann flux is a polynomial function of the edge coordinate and is never evaluated at specific points. Thus, boundary conditions are applied to each component of the flux polynomial, rather than to the flux at specific points.

Boundary conditions can be imposed either by providing the exterior side of the Riemann flux with a complete solution or by reformulating the boundary flux subject to the specified boundary conditions such that only the interior data is needed. However, either approach can be expressed exactly in terms of the other when the equations are linear. The first approach seems trivial to implement; however, this approach has the drawback that in most cases the complete solution is not known. Instead, the complete exterior solution must be reconstructed from the given boundary condition data and the interior solution. The work involved in a carefully derived reconstruction procedure is usually equivalent to the work required to evaluate a completely reformulated flux, although the use (or misuse) of simple extrapolation formulas is common. In this work, the approximate Riemann flux on the boundary is replaced by a reformulated boundary flux.

In the following discussion and examples, the linear Euler equations in two dimensions will be used:

$$
U=\left[\begin{array}{c}
\rho-P \\
p \\
u \\
v
\end{array}\right], \quad \vec{F}=\vec{M} U+\left[\begin{array}{c}
0 \\
\vec{V} \\
\hat{\imath} P \\
\hat{\jmath} P
\end{array}\right]
$$

where $\vec{M} \equiv\left[M_{x}, M_{y}\right], \vec{V} \equiv[u, v]$, and $\hat{\imath}$ and $\hat{\jmath}$ are the Cartesian unit vectors $[1,0]$ and $[0,1]$, respectively. The components of $U$ are normalized perturbation quantities from a free-stream condition about which the linearization has been performed. The compo- nent of the flux normal to the boundary is given by

$$
F(U) \equiv \vec{F}(U) \cdot \vec{n}=M_{n} U+\left[\begin{array}{c}
0 \\
V_{n} \\
\alpha P \\
\beta P
\end{array}\right]
$$

where $M_{n}=\vec{M} \cdot \vec{n}, V_{n}=\vec{V} \cdot \vec{n}, \alpha=\hat{\imath} \cdot \vec{n}, \beta=\hat{\jmath} \cdot \vec{n}$, and $\vec{n}=\mathbf{J}^{-1^{\top}} J \overrightarrow{d s}$ is the boundary-normal vector for an arbitrary edge.

\section{Wall Boundary Conditions}

Wall boundary conditions correspond to the case in which $M_{n}=0$ and $V_{n}$ is specified. Both symmetry-plane and hard-wall boundary conditions state that no flow passes through the boundary; thus, $V_{n}=M_{n}=0$. The symmetry-plane boundary condition should be simply a special case of a general, hard-wall boundary condition in which the wall is planar; however, most finite-difference and finite-volume methods must treat the two differently in order to obtain accurate results. With the discontinuous Galerkin method, the treatment of the two is identical because the discontinuous Galerkin method is valid without modification in the element next to the boundary.

A transpiration wall condition is one in which fluid passes through the boundary at a specified rate. An example is a flow in which blowing or suction is applied to a surface. Another example that is relevant to aerocoustic applications occurs when a flow is separated into incident and scatter components and each component is simulated individually. Occasionally, the form of the incident wave is known exactly, so that only the scattered wave needs to be simulated. With these assumptions, the flux through the boundary is given by

$$
F(U)_{\text {wall }}=\left[\begin{array}{c}
0 \\
\widehat{V}_{n} \\
\alpha P \\
\beta P
\end{array}\right]
$$

The flux is evaluated by using the pressure from the interior element and a specified function for $\widehat{V}_{n}$. The function for $\widehat{V}_{n}$ must be expressed as a polynomial of the edge coordinates. This expression can be obtained by either a Taylor's expansion or a projection procedure. Because the solution within each element is a known polynomial function, the interior solution at the edge is always available without the use of extrapolation formulas.

Figure 1 illustrates a simple application of wall boundary conditions. An acoustic pulse is generated by a pressure disturbance in the initial condition of 
an otherwise undisturbed Mach 0.5 flow. The initial pressure disturbance is a Gaussian distribution with a half-width of 0.05 , centered at $(-0.25,0.25)$ in the domain $0<x, y<1$. A hard-wall condition is specified on the $y=0$ boundary, and through-flow boundary conditions (to be discussed later) are specified on the other three sides of the domain. The results shown are for a discontinuous Galerkin method with $n=4$ (fifth order) and with the domain partitioned by an $18 \times 18$ triangulated grid. At $t=0.4$, the incident pulse has reached the lower wall and has produced a reflection. In this case, the hard-wall boundary is equivalent to a symmetry plane. Figure 1(b) shows similar results in which the computation included the mirror image of the original computational domain. The maximum difference between the solutions is less that 0.1 percent and is attributed to the treatment of the flux at $y=0$. In the first case in which $y=0$ is a wall, the flux at $y=0$ is given by $F(U)=[0,0,0, \beta P]^{T}$. In the case for which the $y=0$ line is within the domain, the flux is evaluated by using the Lax-Friedrichs flux as the approximate Riemann solver

$$
\mathcal{F}\left(U_{u}, U_{l}\right) \equiv\left[F\left(U_{u}\right)+F\left(U_{l}\right)-\lambda\left(U_{u}-U_{l}\right)\right] / 2
$$

where subscripts $u$ and $l$ denote the upper and lower sides of the flux and $\lambda$ is greater than or equal to the magnitude of the largest eigenvalue of $\frac{\partial F}{\partial U}$. Assuming that the solution above and below $y=0$ evolve symmetrically, $U_{u}$ is the same as $U_{l}$ except for the $v$ component, which is an odd function of $y$. Thus, the flux at $y=0$ becomes

$$
\mathcal{F}\left(U_{u}, U_{l}\right)=\left[0,0,0, \beta P_{u}+\lambda v_{u}\right]
$$

Because of the symmetry of the solution and the convergence properties of the discontinuous Galerkin method, $v_{u}$ goes to zero at the rate of $\Delta x^{n+1}$; thus, both formulas are accurate representations of the flux and exhibit the expected convergence properties as the mesh is refined. Note, however, that a low-order error may be introduced if the solution is not symmetric, and if wall boundary conditions are implemented by retaining the approximate Riemann flux and evaluating the exterior solution with a reflection of the interior solution (as is commonly done on low-order finite-volume methods.) The specific form of the error depends on the form of the approximate Riemann solver.

\section{Conditions at Curved Walls}

Walls that are smoothly curved can be modeled with at least second-order accuracy by straight line segments. To improve the accuracy requires a few simple modifications to the im slementation described previously. The first chan e is to compute distinct matrices $\mathbf{M}^{-1} \overrightarrow{\mathbf{A}}$ and $\mathbf{M}^{-1} \mathbf{B}_{k}$ for each element and each side of that eleme it that lies on a curved boundary. The only other hange is simply to recognize that the edge normal vector $\overrightarrow{d s}$ is now a polynomial function instead of a constant vector; thus, $\alpha P$ and $\beta P$ in equation (6) are products of polynomials. Illustrated for triangles in figure 2 , a general triangle with one curver side is mapped (with constant Jacobian) to a simple regular triangle in which the deviation of one si te from its usual straight line path is approximated by a polynomial $\eta(\xi)_{\text {wall }}$. Because the Jacobian $\mathbf{J} \mathrm{i}$. constant within the element, it can be taken out ide the integral; thus all integrations, matrix invercons, and matrix multiplications can be done in advance of the simulation as in the usual implementat on. The primary overhead associated with a curved lement is the additional storage required to store : distinct copy of the matrices for each curved elemt: 1 t.

Figure 3 shows two solutions in hich an acoustic pulse has passed over a cylinder o produce a reflection. In the extreme case show: the cylinder is modeled with only two elements. In figure $3(\mathrm{~b})$ the curved sides are approximated by c bic polynomials. In this test case, the cylinder has a radius of $1 / 2$, and the incident pulse is produce 1 by a Gaussian pressure disturbance in the initial : olution at $x=3$, $y=0$. This case is similar to problem 2 of Category I of the recent workshop The second Workshop on Benchmark Problems for $C A A{ }^{13}$ except that the Gaussian half-width of the initial disturbance has been increased to 1.6 ( 8 times la ger) so that the incident pulse is well resolved on xtremely coarse grids and the error is dominated by the resolution of the cylinder. Figure 4 gives the co:vergence history of the solution as the resolution $i$-increased. The average length scale of an element is defined as

$$
\Delta s=\sqrt{\frac{\text { area of dom }}{\text { number of elei tin }}} \cdot \overline{\text { ients }}
$$

and the error is measured relative o reference solution computed on a fine grid $(\Delta s=0.0498)$. The error is defined as the the $L_{2}$ norm: of the difference in pressure at a large number of points uniformly distributed in the region $0.63<r<2.0,0<\theta<\pi / 2$. The case with the cubic approxim ttion for the wall maintains a fifth-order rate of convergence over the range of grids tested. The rate of convergence for the case with the linear approxim ition for the wall drops to less then third order as tl e mesh is refined. 


\section{Inflow and Outflow Boundary Conditions}

Inflow, outflow, and nonreflective boundary conditions are often treated as different entities; however, for any system of equations such as the Euler equations at subsonic conditions, characteristic information simultaneously enters and leaves the domain through these boundaries. Typically, inflow and outflow boundary conditions have concentrated on ensuring that the correct information enters the domain; nonreflective boundary conditions have concentrated on ensuring that waves that are leaving the domain can do so as if the boundary did not exist.

The simplest form of an inflow and outflow boundary condition is obtained by splitting the flux into characteristic components and grouping the components according to whether their associated wave is entering or leaving the domain. The splitting is given by

$$
\begin{aligned}
F & =\mathbf{P}[\lambda] \mathbf{P}^{-1} U \\
& =\mathbf{P}\left[\lambda^{+}\right] \mathbf{P}^{-1} U_{\text {interior }}+\mathbf{P}\left[\lambda^{-}\right] \mathbf{P}^{-1} U_{\text {exterior }}
\end{aligned}
$$

where $\mathbf{P}$ and $[\lambda]$ are the eigenvector matrix and the diagonal matrix composed of the eigenvalues of $\frac{\partial F}{\partial U}$ respectively, and $\left[\lambda^{ \pm}\right]$are diagonal matrices composed of just the positive or negative elements of $[\lambda]$. The exterior solution is usually set to zero; however, the solution could be set to any desired value to accommodate the case in which a specified wave is to enter the domain. This approach has been used in the results shown thus far and provides a crude, nonreflective boundary condition in that waves that are nearly aligned with the boundary will exit with little reflection. The method of Thompson ${ }^{19}$ is an analogous procedure formulated for finite-difference methods.

The reason for the reflection is that when an outgoing wave that is not aligned with the boundary is decomposed into boundary-normal and boundarytangent characteristic components, the inbound boundary-normal characteristic component is not exactly 0 . Yet in almost all characteristic-based boundary conditions the inbound boundary-normal component set set either to 0 or to some specified value that has no relation to any outgoing wave that might exist. Most attempts to improve the nonreflective boundary condition involve derivation of a means to reconstruct an inbound boundary-normal characteristic contribution associated with outgoing waves. The most accurate of these methods $7,9,11$ involves the solution of an additional partial differential equation along the boundary. Thus far, these boundary conditions have only been formally derived for smooth (if not planar) boundaries for which the mean flow is strictly inflow or outflow over the entire boundary.

Another approach, the finite-wave model, ${ }^{20}$ provides a simple (algebraic) method for improving the accuracy in some cases. This boundary condition was developed to deal with nonlinear effects of the Euler equations; however, the method also accounts for wave orientation in a way that is applicable to the linear case. The linear analog of the finite-wave mode is a simple modification to the standard characteristic approach and will be referred to as the modified characteristic method. The directionality inherent in the usual characteristic splitting arises because the boundary flux is the flux in the direction of the boundary normal. The direction associated with the flux cannot be altered; however, characteristic decomposition could certainly be based on another direction. In fact, because the boundary of the domain may not have any relation to the sound produced within the domain, other directions should be considered for the characteristic decomposition. If a single identifiable acoustic source is assumed, then the finite-wave model performs a characteristic decomposition along the assumed path of the wave. The decomposition is obtained from the characteristic variables associated with the flux in a prescribed direction:

$$
F_{w}(U) \equiv \vec{F}(U) \cdot \vec{w}=M_{w} U+\left[\begin{array}{c}
0 \\
V_{w} \\
\alpha_{w} P \\
\beta_{w} P
\end{array}\right]
$$

where $\vec{w}$ is a unit vector in a prescribed direction and other quantities are defined as in equation (5) with $\vec{n}$ replaced by $\vec{w}$. The solution at the boundary associated with waves that are leaving the domain in the direction of $\vec{w}$ is given by $U_{b}=\mathbf{P}_{\mathbf{w}}\left[\mathbf{I}^{+}\right] \mathbf{P}_{\mathbf{w}}^{-1} U_{\text {interior, }}$, where $\mathbf{P}_{\mathbf{w}}$ are the eigenvectors of $\frac{\partial F_{w}}{\partial U}$ and $\left[I^{+}\right]$is a diagonal matrix with elements equal to 1 if the corresponding eigenvalue of $\frac{\partial F_{w}}{\partial U}$ is positive and equal to 0 otherwise. The flux through the boundary is given by evaluating equation (5) with the solution $U_{b}$.

The standard and modified characteristic methods are compared in figure 5 . The test case is the cylinder problem previously described with the nonreflecting boundary conditions imposed at $r \approx 5.3$. At time $t=10$ most of the physical waves have exited the domain, and the remaining disturbances are caused by unwanted reflections. The modified characteristic boundary condition has reduced the reflection to less than half that of the standard characteristic boundary condition but the general form of the reflection is unchanged. 


\section{The PML Method}

The PML method is a buffer-zone technique that solves a modified set of equations in a region that surrounds the primary computational domain. The modified equations are obtained by splitting the equations in boundary-normal and boundarytangent directions and adding low-order damping to the boundary normal equations. For example, at boundaries aligned with either $\hat{\imath}$ or $\hat{j}$,

$$
\frac{\partial U_{1}}{\partial t}+\frac{\partial(\hat{\imath} \cdot \vec{F}(U))}{\partial x}=-\sigma_{x} U_{1}
$$

and

$$
\frac{\partial U_{2}}{\partial t}+\frac{\partial(\hat{\jmath} \cdot \vec{F}(U))}{\partial y}=-\sigma_{y} U_{2}
$$

where $U=U_{1}+U_{2}$. The damping coefficients $\sigma_{x}$ and $\sigma_{y}$ must be chosen such that the component of $\sigma$ that is tangent to the boundary does not vary along the boundary. This condition leads to the constraint on $\sigma$ in corner regions illustrated in figure 6 . Research has shown that, ${ }^{10}$ for the ideal case of plane waves and straight boundaries that intersect at right angles (i.e., rectangular domains), no reflection of acoustic or convective waves will occur at the interface between the primary computational domain and the PML zone, regardless of the angle at which waves strike the interface. The underlying theory places no constraint on the variation of $\sigma$ in the direction normal to the boundary, but in applications to finitedifference methods $\sigma$ must vary smoothly. In numerical tests by $\mathrm{Hu},{ }^{10}$ the boundary-normal component of $\sigma$ was increased quadratically as a function of the distance from the interface.

When the PML method is applied to the discontinuous Galerkin method, $\sigma$ does not need to be varied smoothly. Furthermore, using a constant value of $\sigma$ throughout a PML zone is advantageous. In the present application of the PML method to the discontinuous Galerkin method, the equations are solved in a different, but equivalent, form. In PML zones that border on the physical domain, the sum of the two split equations is solved in combination with the boundary-tangent equation. For example, on a boundary where $x=$ Constant, $\sigma_{y}=0$ and the equations can be rewritten as

$$
\frac{\partial U}{\partial t}+\nabla \cdot \vec{F}(U)=-\sigma_{x}\left(U-U_{2}\right)
$$

and

$$
\frac{\partial U_{2}}{\partial t}+\frac{\partial(\hat{\jmath} \cdot \vec{F}(U))}{\partial y}=0
$$

The first equation is the standard nterior operator modified only by a zeroth-order issipation term; thus, this equation is easily implen ented within the existing program structure. In a curner region, the sum of the split equations is solve $I$ in conjunction with either equation (7) or (8):

$$
\frac{\partial U}{\partial t}+\nabla \cdot \vec{F}(U)=-\sigma_{x} U+\left(\sigma_{x}-\sigma_{y}\right) U_{2}
$$

and

$$
\frac{\partial U_{2}}{\partial t}+\frac{\partial(\hat{\jmath} \cdot \vec{F}(U))}{\partial y}=-\sigma_{y} U_{2}
$$

Note, however, that if $\sigma_{x}=\sigma_{y}=$ Constant throughout the corner region, then the in dividual components $U_{1}$ or $U_{2}$ do not contribute o equation (11); thus, only equation (11) needs to Le solved.

In figures 7 and 8 , solutions of tained with the PML method are compared with those obtained with characteristic boundary conditions. The test problem is a square domain $(-50 \therefore x, y<50)$ with hard-wall boundary conditions apllied on the top, bottom, and left boundaries and either a PML zone or a characteristic boundary condition applied at the right boundary. The unsteady flo $w$ is initiated by a unit Gaussian pressure disturbence with a halfwidth of 3 , positioned at $x=25, y=0$. The primary domain is partitioned with a $18 \times 18$ triangulated grid; the PML case has two layers of elements in which the values of $\sigma$ are constant: $\sigma_{x}=0.2$ and $\sigma_{y}=0$. The solutions are compared with a baseline case in which the right boundary is extended to $x \approx 161$. Figure 7 shows the solıtions at $t=40$, which is just after the initial pulse has reached the boundary. The solution obtained with characteristic boundary conditions has weak refiections, and the solution obtain with the PML mtthod agrees well with the baseline. Figure 8 show the solutions at a much later time $(t=180)$ when eflections off the solid walls have produced a complex wave pattern. The solution obtained by using the PML method still agrees well with the baselin- solution, while the standard characteristic method shows additional features that can only be attribut $\mathrm{d}$ to nonphysical reflections off the right boundary.

Figure 9 shows the effect of inc easing the thickness of the PML layer $\left(x_{b}-50\right)$ and varying the values of $\sigma_{x}$. The error metric is the maximum deviation of pressure from that of the baseline solution measured along the line $x=48$ for $t<200$. The solid line denotes the case in which $\sigma_{x}$ was varied quadratically, as described in reference 10 ; the dashed lines denote cases in which $\sigma_{x}$ is fixed at one of several values. Note that the $\mathrm{d}$ ta at $x_{b}=50$ re- 
sult from use of the standard characteristic boundary condition.

Finally, the PML method is applied to the cylinder problem show earlier in the region $r>5.3$. Figure 10 shows the maximum pressure difference from the baseline solution measured at $r=5, t<12$. In this case, the PML method is implemented by assigning a normal direction to each element in the PML zone. Even though the boundary is curved and the normal direction varies in each element, the PML method shows a considerable improvement over the modified characteristic method $\left(r_{b} \approx 5.3\right)$.

\section{Concluding Remarks}

The application of several types of boundary conditions for the discontinuous Galerkin method is presented. Because of the compact form of the method, the discontinuous Galerkin method is applicable near boundaries without modification; this feature eliminates a major difficulty encountered by most high-order methods. As a consequence, boundary conditions such as symmetry-plane, curved-wall, and characteristic inflow outflow, are easy to implement and highly accurate. With modified characteristic boundary conditions that account for the direction of wave propagation, reflections are reduced to about half that of the standard characteristic method. The perfectly matched layer (PML) method is easily applied to the discontinuous Galerkin method. The discontinuous Galerkin method allows the damping parameters to be abruptly turned on and then held constant within the PML zone. Reflections are reduced by an order of magnitude below that of characteristic boundary conditions, even in cases with curved boundaries.

\section{References}

1. H. L. Atkins and Chi-Wang Shu, "QuadratureFree Implementation of the Discontinuous Galerkin Method for Hyperbolic Equations", AIAA Paper 96-1683, 1996.

2. Christopher K. W. Tam and J. C. Webb, "Dispersion-Relation-Preserving Finite Difference Schemes for Computational Aeroacoustics," Journal of Computational Physics, Vol. 107, 1993, pp. 262-281.

3. Christopher K. W. Tam and Zhong Dong, "Wall Boundary Conditions for High-Order Finite-Difference Schemes in Computational Aeroacoustics," Theoret. Comput. Fluid Dynamics, Vol. 6, 1994, pp. 303-322.
4. A. Jameson, W. Schmidt, and E. Turkel, "Finite Volume Methods Using Runge-Kutta Time-Stepping Schemes," AIAA Paper 811259,1981 .

5. A. Verhoff, D. Stookesberry, and S. Agrawal "Far-Field Computational Boundary Conditions for Two-Dimensional External Flow Problems," AIAA Journal, Vol. 30, 1992, pp. 25852594 .

6. S. V. Tsynkov, "Artificial Boundary Conditions for Computation of Oscillating External Flows," NASA-TM-4714, 1996/

7. Engquist, Bjorn and Majda, Andrew, "Absorbing Boundary Conditions for the Numerical Simulations of Waves," Mathematics of Computation, Vol. 31, 1977, pp. 629-651.

8. T. Hagstrom, "Boundary Conditions at Outflow for problems with transport and Diffusion," J. Comp. Phys., Vol. 69, 1987, pp. 69-80.

9. Michael B. Giles, "Non-Reflecting Boundary Conditions for Euler Equation Calculations" AIAA Paper 89-1942, 1989.

10. Fang $\mathrm{Q} . \mathrm{Hu}$, "On Absorbing Boundary Conditions for Linearized Euler Equations by Perfectly Matched Layer," NASA CR-198224, 1995.

11. John W. Goodrich and Thomas Hagstrom, "Accurate Algorithms and Radiation Boundary Conditions for Linearized Euler Equations," AIAA Paper 96-1660, 1996.

12. B. Cockburn and C.-W. Shu "TVB RungeKutta local projection discontinuous Galerkin finite element method for conservation laws II: general framework," Mathematics of Computation, v52 (1989), pp. 411-435.

13. B. Cockburn, S. Hou, and C.-W. Shu "The Runge-Kutta local projection discontinuous Galerkin finite element method for conservation laws IV: the multidimensional case," Mathematics of Computation, v54 (1990), pp. 545581 .

14. F. Bassi and S. Rebay "Accurate 2D Euler Computations by means of a High-Order Discontinuous Finite Element Method," Proceedings of the 14th international Conference on $\mathrm{Nu}$ merical Methods in Fluid Dynamics, Bangalor, India, July 11-15, 1994. 
15. "A Space-Time Discontinuous Galerkin Method for Time-Accurate Numerical Solution of $\mathrm{Hy}$ perbolic Conservation Laws," presented at the 12th AIAA computational Fluid Dynamics Conference, San Diego, CA, June 19-22, 1995.

16. C.-W. Shu and S. Osher, "Efficient implementation of essentially non-oscillatory shockcapturing schemes," Journal of Computational Physics, v77 (1988), pp. 361-383.

17. B. Gustafson, H.-O. Kreiss, and A. Sundström. "Stability theory of difference approximations for mixed initial-boundary value problems, II." Mathematics of Computation, Vol. 26, 1972, pp. 649-686.

18. Proceedings of the Second Workshop on Benchmark Problems in Computational Aeroacoustics, NASA CP in preparation, 1997.

19. Kevin W. Thompson, "Time Dependent Boundary Conditions for Hyperbolic Systems," Journal of Computational Physics, Vol. 68, 1987, pp. 1-24.

20. H. Atkins and J. Casper "Nonreflective Boundary Conditions for High-Order Methods," AlAA Journal, Vol. 32, 1994, pp. 512-518.

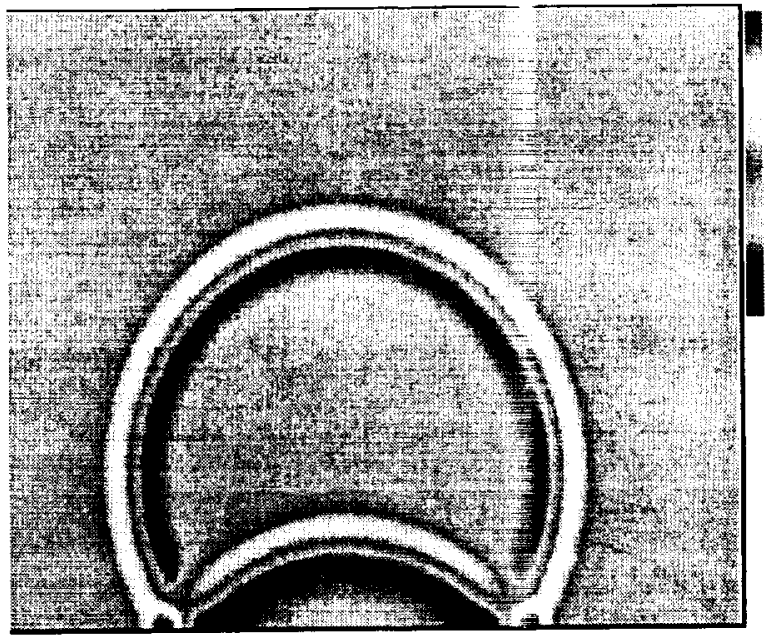

a. Wall condition at $y=0$.

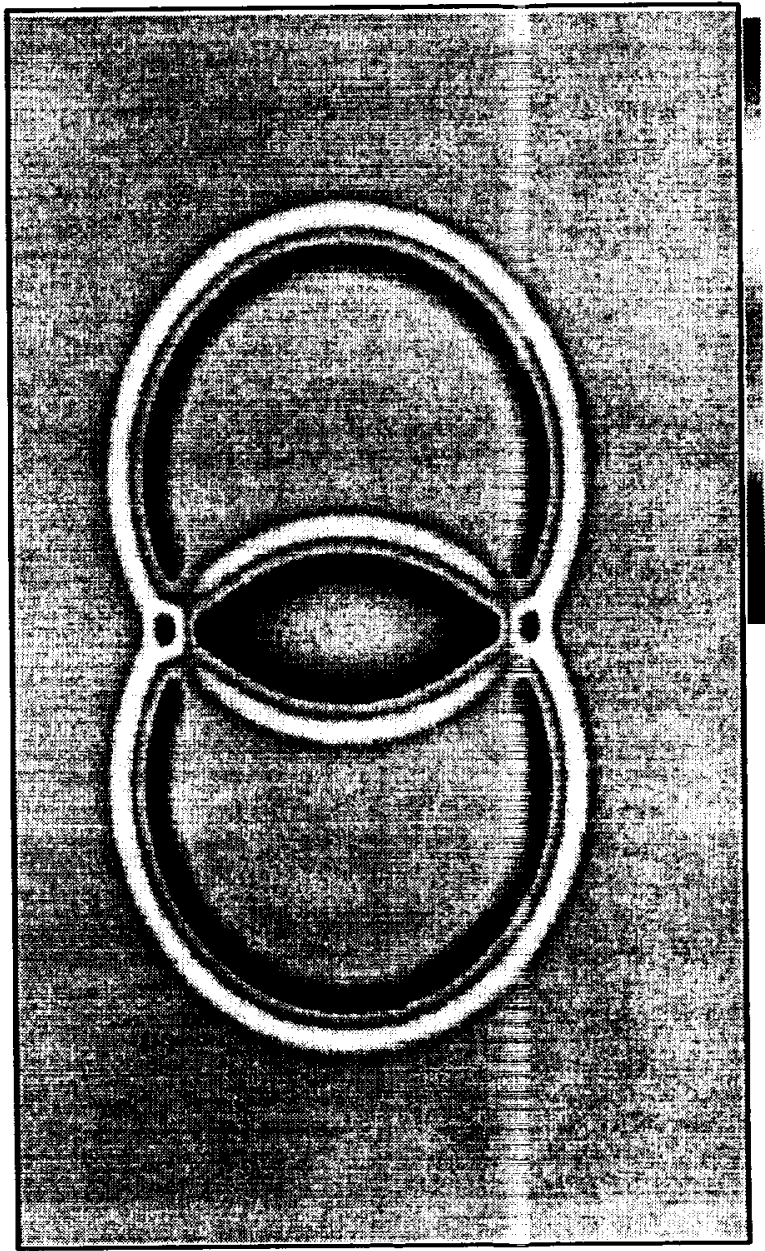

b. Computed mirror image of p:imary domain.

Figure 1. Reflection of cylindrical pressure pulse off a flat wall compared with direct $c$ mputation of primary domain plus mirror image. 


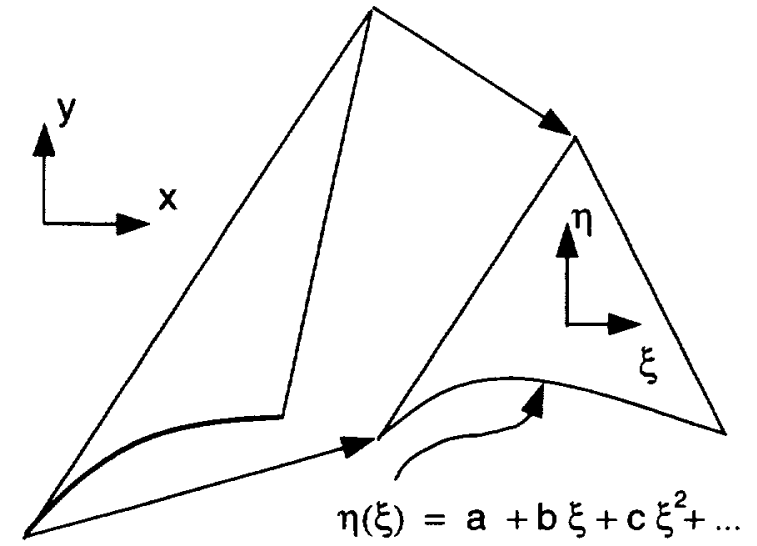

Figure 2. Mapping of curve-wall element.

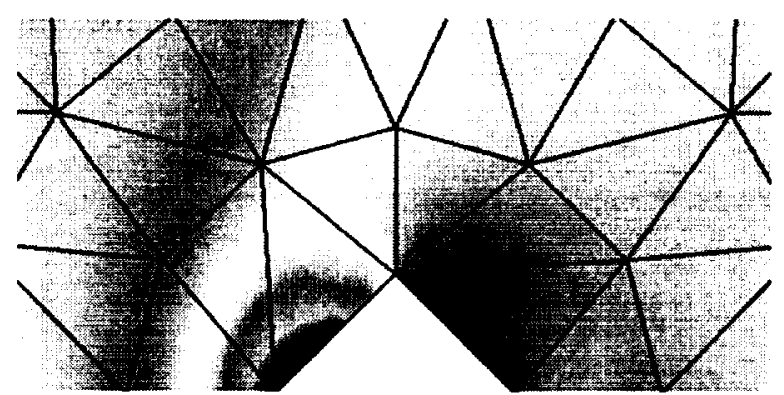

a. Linear wall segments.

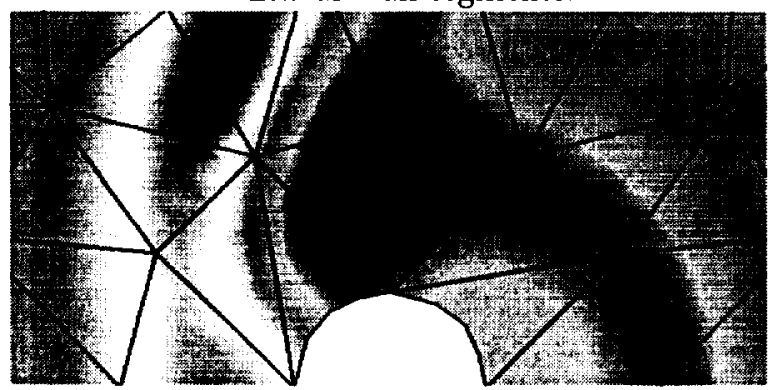

b. Cubic wall segments.

Figure 3. Reflection of cylindrical pressure pulse off of solid cylinder.

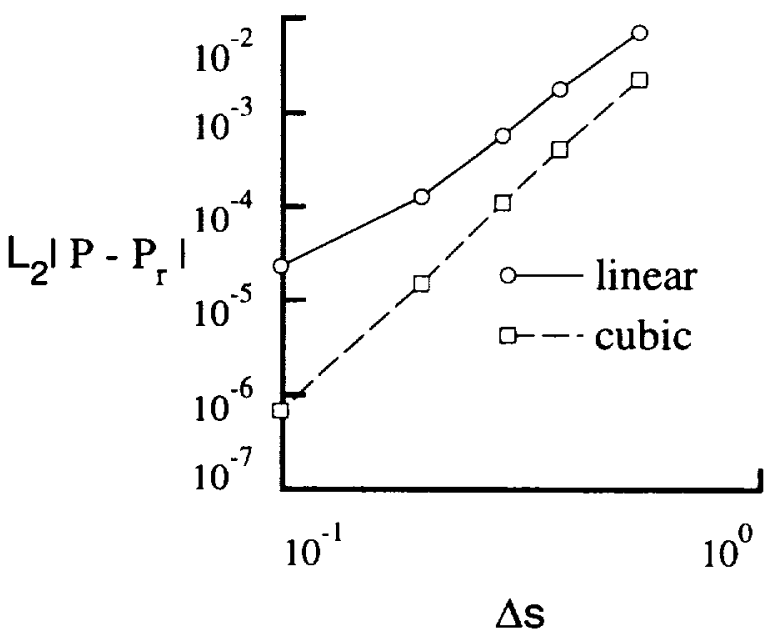

Figure 4. Convergence of solution error with grid refinement.

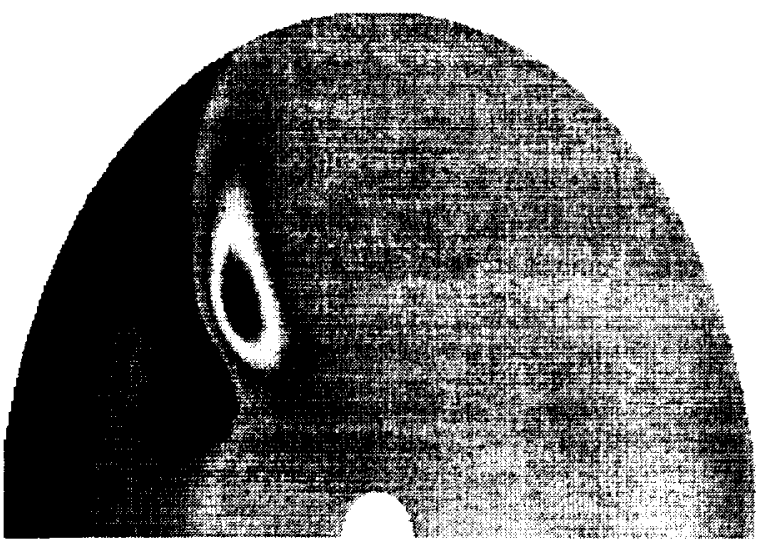

a. Standard characteristic boundary conditions.

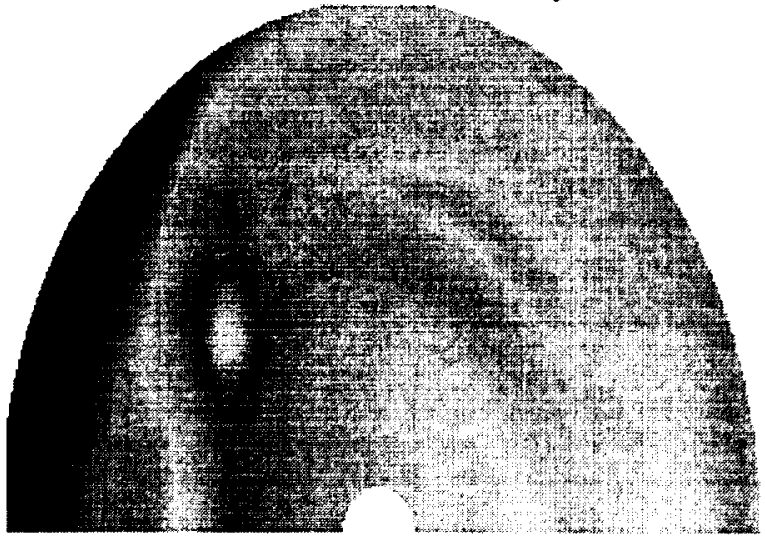

b. Modified characteristic boundary conditions.

$P_{\min }=-0.0914$ $P_{\max }=0.021$

Figure 5. Comparison of nonreflecting boundary conditions applied at $r \approx 5.3$ : pressure at $t=10$. 


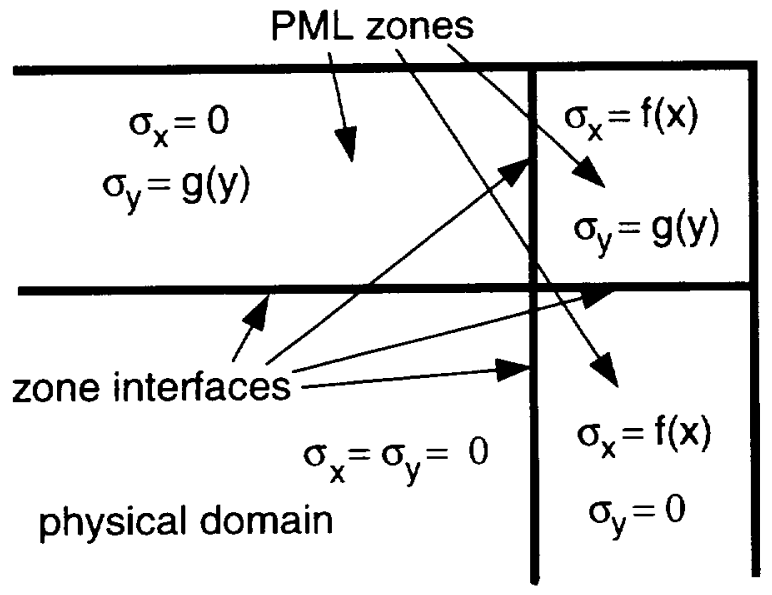

Figure 6. PML zones with consistent values of $\sigma$.

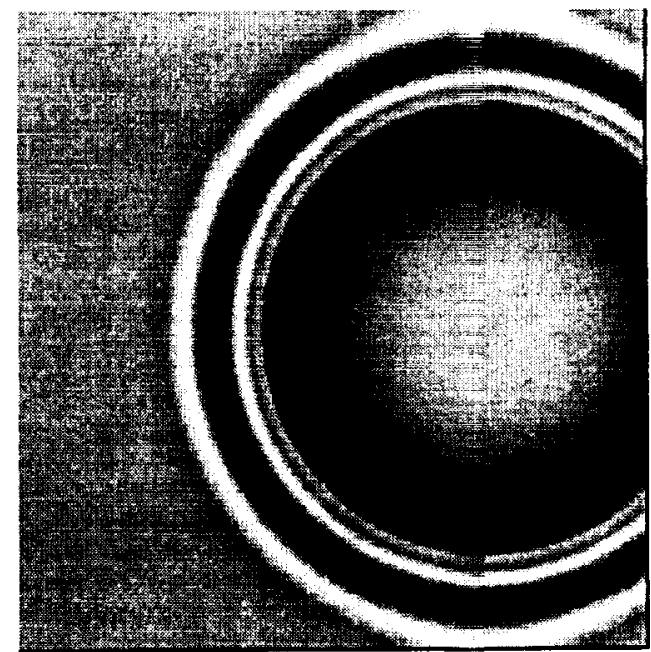

a. Baseline solution.

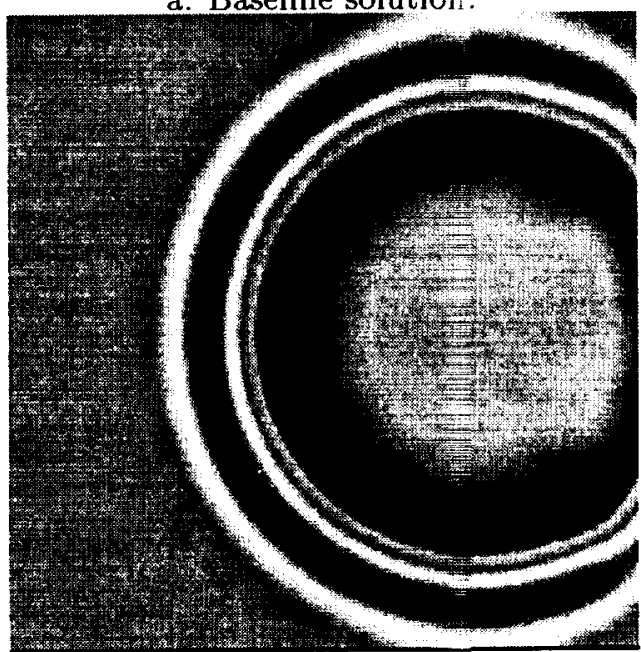

b. Characteristic boundary condition.

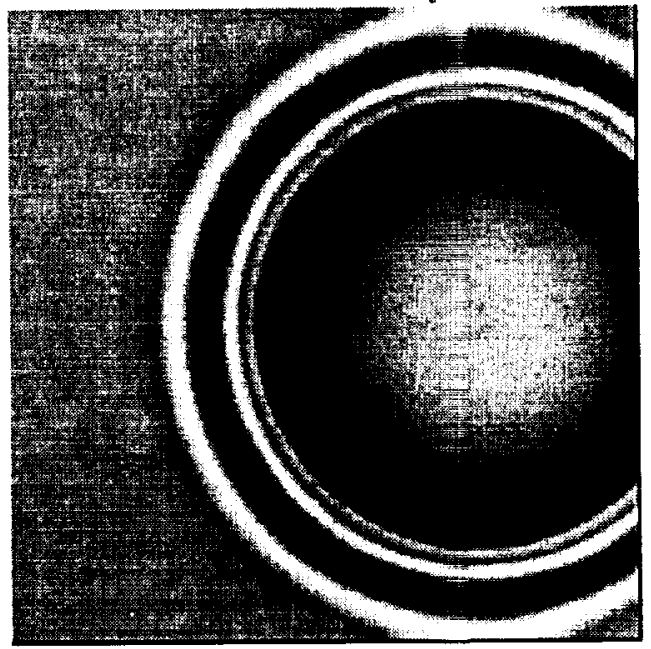

c. PML zone.

Figure 7. Comparison of pressl:e with different treatment of right boundary: $t=0$. 


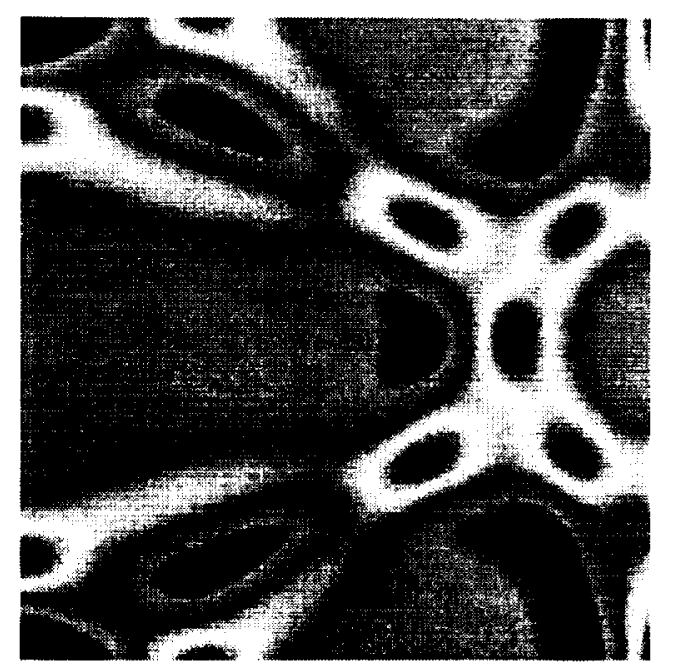

a. Baseline solution.

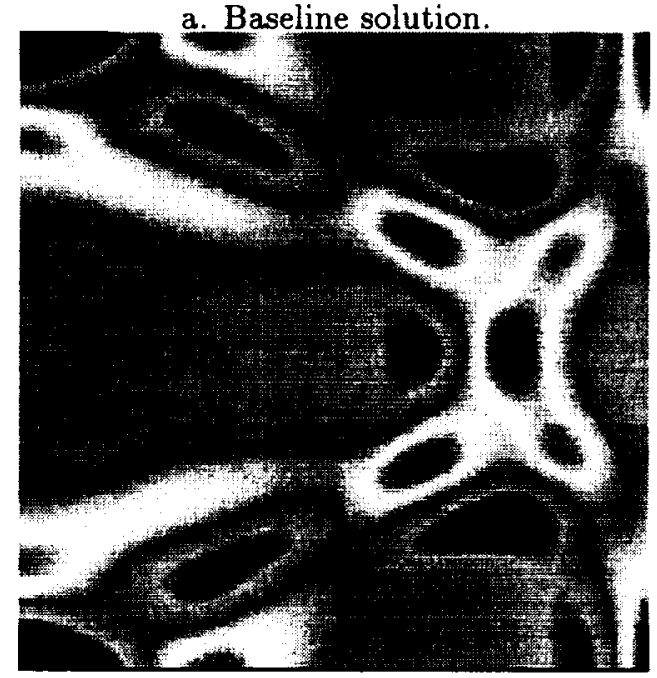

b. Characteristic boundary condition.

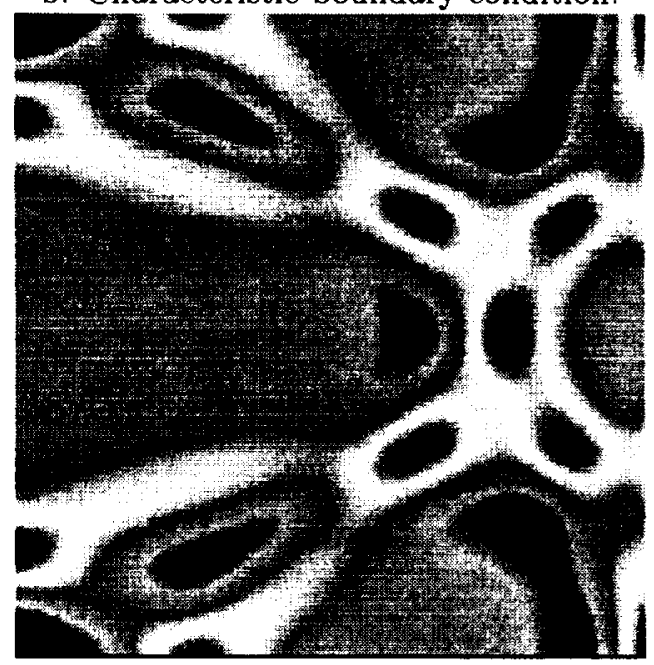

c. PML zone.

Figure 8. Comparison of pressure with different treatment of right boundary: $t=180$.

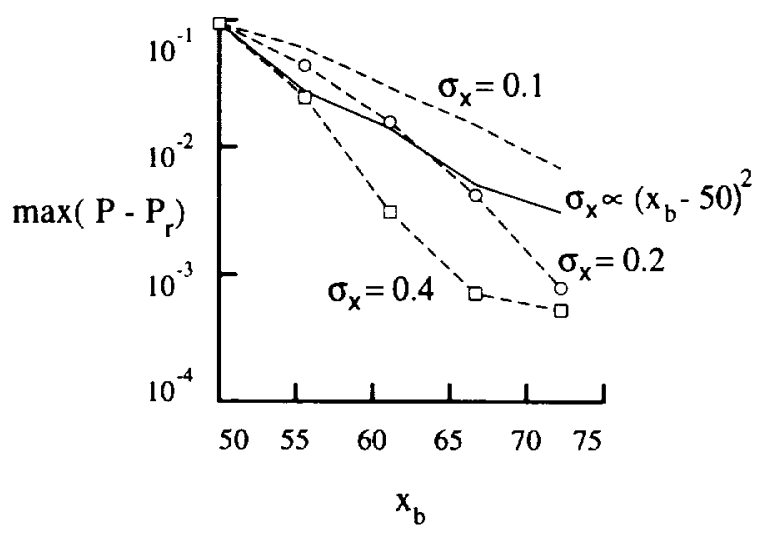

Figure 9. Effect of thickness of PML layer and value of $\sigma$ for flow shown in figures 7 and 8 .

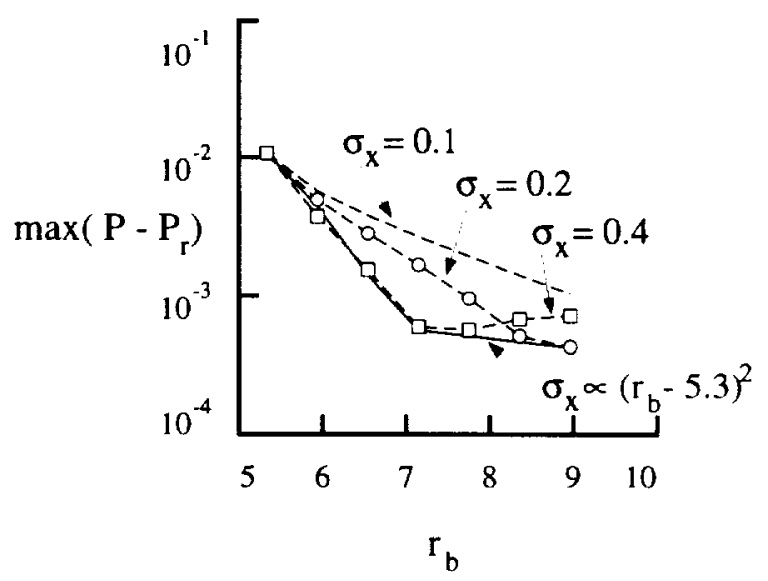

Figure 10. Effect of thickness of PML layer and value of $\sigma$ for flow shown in figure 5 . 